\title{
Autophagy may play an important role in varicocele
}

\author{
SHAO-MING ZHU ${ }^{1 *}$, TING RAO $^{1 *}$, XIAO YANG $^{2}$, JIN-ZHUO NING $^{1}$, WEI-MING YU ${ }^{1}$, YUAN RUAN $^{1}$, \\ RUN YUAN $^{1}$, CHENG-LONG LI ${ }^{1}$, KUN JIANG ${ }^{1}$, WEI HU ${ }^{1}$, HAO-YONG LI ${ }^{1}$ and FAN CHENG ${ }^{1}$ \\ Departments of ${ }^{1}$ Urology, and ${ }^{2}$ Gynaecology and Obstetrics, Renmin Hospital of Wuhan University, \\ Wuhan, Hubei 430060, P.R. China
}

Received February 19, 2017; Accepted August 4, 2017

DOI: $10.3892 / \mathrm{mmr} .2017 .7253$

\begin{abstract}
The present study aimed to determine the expression of autophagy and investigate whether the hypoxia-inducible factor $1 \alpha(\mathrm{HIF}-1 \alpha) / \mathrm{BCL} 2$ interacting protein (BNIP3)/Beclin-1 autophagy signaling pathway serves an important role in activating autophagy in varicocele (VC) rat testes cells. Furthermore, the current study aimed to explain the possible association between autophagy and apoptosis. A total of 48 adult male Sprague Dawley rats were divided into group A (control), group B (VC 15-day), group C (VC 30-day) and group D (VC 45-day), with 12 rats in each group. The rats in group A did not receive any interventions, and in groups $\mathrm{B}, \mathrm{C}$, and D the VC model was established simultaneously. At $0,15,30$, and 45 days, an orchidectomy on the left testes was performed in groups A-D, each on its respective day. Transmission electron microscopy was used to investigate the expression of autophagy. Compared with groups A and B, it was demonstrated that the expression of autophagy in groups $\mathrm{C}$, and D was significantly increased. Hematoxylin and eosin staining revealed that as the rats survived $\mathrm{VC}$ longer, the testicular tissue damage became more serious. Furthermore, the Johnson score revealed that VC impaired the spermeiogenesis function of the male rats. Additionally, it was demonstrated that the apoptosis index of the seminiferous epithelia cells in $\mathrm{VC}$ rat testes increased over time, as measured using TUNEL staining. Immunohistochemical analysis revealed that as the $\mathrm{VC}$ was prolonged, the expression of HIF-1 $\alpha$ gradually increased while the expression of (apoptosis regulator $\mathrm{Bcl}-2$ ) $\mathrm{Bcl}-2$ gradually decreased. Furthermore, western blot analysis revealed that the protein expression of $\mathrm{Bcl}-2$ decreased and apoptosis regulator Bax increased. Furthermore, HIF-1 $\alpha$, BNIP3, Beclin1 and microtubule associated protein 1 light chain $3 \alpha$ (LC3)II/LC3I expression gradually increased. However, significant increases
\end{abstract}

Correspondence to: Dr Fan Cheng, Department of Urology, Renmin Hospital of Wuhan University, 9 Zhangzhidong Road, Wuhan, Hubei 430060, P.R. China

E-mail: urology1969@aliyun.com

${ }^{*}$ Contributed equally

Key words: autophagy, varicocele, apoptosis, testis, HIF-1 $\alpha$, BNIP3, Beclin1 in Beclin 1 and LC3II/LC3I were only observed between the day 0 and day 30 groups. In addition, the expression of p62 significantly increased between day 0 and day 15 , but gradually decreased between day 15 and day 45 . The results of the present study revealed that $\mathrm{VC}$ can lead to testicular tissue hypoxia, and that the HIF-1 $\alpha$ /BNIP3/Beclin1 autophagy signaling pathway may upregulate autophagy in VC rats testes. Thus, the association between autophagy and apoptosis may serve an important role in male infertility caused by VC.

\section{Introduction}

Varicocele is an abnormal venous dilatation and/or tortuosity of the pampiniform plexus in the scrotum. VC is one of the main factors causing male infertility, and male patients with VC account for approximately $15 \%$ of all healthy male adults and approximately 35\% of patients with primary infertility (1). VC causes severe damage to male reproductive health. Although the high ligation of the spermatic vein is a good way to improve a patient's semen quality, there are still some patients unable to restore fertility, and studies have found that the surgery cannot improve the postoperative natural pregnancy rate $(2,3)$. Therefore, it is particularly important to clarify the mechanism of $\mathrm{VC}$ leading to male infertility; unfortunately, the mechanism is not yet fully clear.

The hypoxia-inducible transcription factors (HIFs) are induced under hypoxia, and they mainly comprise HIF-1 and Hif-2. HIF-1 comprises two subunits, HIF-1 $\alpha$ and HIF-1 $\beta$. HIF-1 $\beta$, as a stable subunit, could express stably in hypoxic cells. HIF-1 $\alpha$ is often selected as a biomarker of hypoxia (4). HIF- $1 \alpha$, as the activity subunit of HIF-1, is regulated by the oxygen signal, which controls the activity of Hif1. HIF-1 $\alpha$ transfers to the nucleus to activate a series of genes that affect cell metabolism, growth, proliferation, biosynthesis, apoptosis, and autophagy $(5,6)$. One of the important roles of HIF-1 $\alpha$ is to activate the BNIP3 (Bcl-2 and adenovirus E1B 19-kDa interacting protein 3) genes, which leads to the synthesis of more BNIP3 protein (7). As one of the Bcl-2 family proteins, BNIP3 contains BH3 Domains. The HIF-1 $\alpha /$ beclin-1 signal pathway plays an important role in the activation of autophagy $(8,9)$. Although studies have found that HIF-1 $\alpha$ was significantly increased in $\mathrm{VC}$ rat testes $(10,11)$, the role of HIF-1 $\alpha$ in VC is still unclear.

The autophagosome, induced by cell hunger, hypoxia, or infection, endocytoses cytoplasm, damaged organelles, and redundant proteins and then fuses with the lysosome, 
eventually forming an autolysosome. The contents are hydrolyzed in the autolysosome to utilize the energy, amino acids, and other cellular components. Autophagy and apoptosis are often inseparable and highly interactional. In different diseases, the relationship between autophagy and apoptosis is not the same. Wu et al (12) found that specifically blocking autophagy enabled ROS to increase significantly in malignant glioma cells; thus, autophagy can aggravate the apoptosis of tumor cells. Another study found that autophagy could improve the ability of cells to fight infection, which reduces the apoptosis of infected cells (13). However, the promotion of autophagy is also increased during cell apoptosis (14). Taking all of the above into consideration, what promotes autophagy in $\mathrm{VC}$ that inhibits apoptosis or what promotes apoptosis is unknown.

Apoptosis is the main method of programmed cell death. Apoptosis is mainly mediated by two pathways: the first is via a membrane receptor, such as Fas/FasL; the second is the mitochondrial pathway (15). The latter mainly regulates the MOMP (mitochondrial outer membrane permeabilization) through the Bcl-2 family proteins. The Bcl-2 family contains many proteins, such as the proapoptotic proteins Bax and BAK, the antiapoptotic proteins Bcl-2, Bcl-xL, and BH3-the only protein containing $\mathrm{BH} 3$ domains (16). Under normal conditions, the antiapoptotic protein's hydrophobic groove combines with the $\mathrm{BH} 3$ domains of $\mathrm{BH} 3$ s-only protein and antiapoptotic proteins, which can form heterodimers to prevent MOMP. In addition, Bcl-2 can also function as an antioxidant, clearing reactive oxygen species (ROS). Bcl-2 also affects transmembrane transport, changes the distribution of calcium ion, and activates endogenous enzymes $(17,18)$.

When under the condition of hypoxia, infection, or oxidative stress, the apoptosis pathway is activated. Proapoptotic proteins translocate to the mitochondria, causing damage to its structure and function, causing MOMP. In addition, the caspases and other hydrolases are released into the cytoplasm, which causes irreversible damage to the cells. The apoptosis of seminiferous epithelia cells affects the quality and quantity of sperms, and it may be an important reason for male infertility caused by VC. Some studies found that the apoptosis of spermatogenic cells significantly increased in VC rat testes $(19,20)$. In addition, other studies found that the expression of the Bax gene and protein is increased in human semen $(21,22)$.

The present study mainly focuses on the observation of autophagy and apoptosis in VC rat testes, tries to verify whether the HIF-1 $\alpha /$ BNIP3/Beclin1 autophagy signaling pathway is highly expressed, and provides a brief analysis about the possible relation between autophagy and apoptosis in VC rat testes.

\section{Materials and methods}

Animal study. The experimental procedures and the animal use and care protocols were approved by the Committee on Ethical Use of Animals of Renmin Hospital of Wuhan University. In all, 48 adult SD male rats (approximately 200-240 g) were purchased from the Experimental Animal Center of The Centers for Disease Control (Wuhan, China) and randomized to 4 groups: Group A (control group/VC 0 days), group B (VC 15 days), group C (VC 30 days), group D
(VC 45 days), with 12 rats in each group. All rats were fed in the same SPF environment, and all operations were performed under aseptic conditions. Each rat was raised for three days in the same environment after purchase to allow adaptation to the new environment. All rats in group A were anesthetized and decapitated to obtain the left testis. Partial ligation of the left renal vein surgery was used to establish the VC model. Each rat in groups B, C, and D underwent this surgery under the same conditions at the same time. The left testis of groups B, $\mathrm{C}$, and D were obtained 15, 30, and 45 days after establishing the VC model, respectively.

VC model creation. According to the method of Turner (23), $1 \%$ pentobarbital sodium $(35 \mathrm{mg} / \mathrm{kg}$ ) was administered via intraperitoneal injection to induce anesthesia. After anesthesia was achieved, we fixed the limbs and chose an abdominal median incision. We opened the abdominal cavity, moved the viscera around the left kidney to the right, and then completely exposed the left kidney, left renal vein, left spermatic vein and inferior vena cava. We isolated the left renal vein at the junction of the left renal vein and inferior vena cava and then placed a $0.8-\mathrm{mm}$ metal bar under the left renal vein. Then, 3-0 silk was used to ligate the left renal vein and metal bar at the junction. This reduced the diameter of the left renal vein by half. Then, we drew out the metal rods and immediately observed the expansion of the left renal vein. The branch veins of the spermatic vein were also ligated. After peritoneal lavage with normal saline, we sutured the abdomen with 4-0 silk suture. Success criteria: The size was not obviously different between left and right sides of the kidneys. However, 1 rat of group C and 2 rats of group D were found with the left kidney obviously atrophied, therefore they were excluded from the study. Consequently, the number of the samples in each group was $12,12,11,10$.

Antibodies. Rabbit anti Hif1 $\alpha$ primary antibody, sc-10790, was obtained from Santa Cruz Biotechnology, Inc. (Dallas, TX, USA). Mouse anti BNIP3 primary antibody, Abcam Ab10433, was purchased from Abcam (Abcam, Cambridge, UK). Rabbit anti Beclin1 primary antibody, CST 3738, was obtained from Cell Signaling Technology (Danvers, MA, USA). Rabbit antibody LC3 (CST 2775) rabbit antibody p62 (CST 5114) and mouse antit Bcl-2 primary antibody, (sc-7382). Rabbit anti Bax (CST 14796) and rabbit anti GAPDH, AB-P-R 001, were provided by Hangzhou Goodhere Biotechnology Co. (LTD, Hangzhou, China). Horseradish peroxidase (HRP) labeled sheep anti IgG secondary antibody, BA1054, were obtained from Wuhan Boster Biological Engineering Co. (Wuhan, China).

Jonhsen score. H\&E staining samples were observed under a 40x10 Olympus microscope (Olympus Corp., Tokyo, Japan) and 10 images in each sample were randomly selected for Jonhsen score (24). To judge spermatogenesis, according to the Jonhsen scores. Johnsen scores ranging between 1 and 10 . While a score of 10 represents an excellent spermatogenesis, 1 represents a worst level.

Transmission electron microscopy (TEM). A small piece of testicular tissue was cut with a sharp blade in $1 \mathrm{~min}$, with a size 
of approximately $0.2 \mathrm{~cm}^{3}$, and fixed in $2.5 \%$ glutaraldehyde. Then, the tissue was sliced into approximately $1 \mathrm{~mm}^{3}$ in fixative at $4^{\circ} \mathrm{C}$ for $12 \mathrm{~h}$. The testicular tissues were washed with $0.1 \mathrm{M}$ phosphate buffer saline buffer, fixed in $1 \%$ osmic acid for $1 \mathrm{~h}$, washed in PBS again, and dehydrated in ethanol, acetone and epoxy resin for $4 \mathrm{~h}$. After being embedded and fixed, the prepared tissue was cut to $60-\mathrm{mm}$ sections and stained with uranyl acetate and lead citrate and then observed under an transmission electron microscope (H-7600; HITACHI, Ltd., Tokyo, Japan). Fifteen fields were randomly selected for each sample.

TUNEL staining. Terminal-deoxynucleoitidyl Transferase Mediated Nick and Labeling (TUNEL) staining was used to detect apoptosis of the spermatogenic cells of the testes. Prepared tissue was fixed with $4 \%$ neutral formaldehyde, embedded in paraffin and serially cut. After regular dewaxing, hydration, and serum blocking, we used an In Situ Cell Death Detection kit, POD (REF no. 11684817910, Roche Diagnostics GmbH, Mannheim, Germany) according to the manufacturer's instructions. The cells were observed and photographed under an optical microscope. Ten high image fields of each sample were randomly selected, and 200 cells were counted in each field. The apoptosis index (AI) was calculated according to the number of apoptotic spermatogenic cells.

Immunohistochemistry. An immunohistochemistry kit was purchased from Dako Denmark A/S (K5007, Code; Glostrup, Denmark). Briefly, paraffin sections were deparaffinized, dehydrated, and then soaked in boiled $0.01 \mathrm{~mol} / 1$ citric acid buffer ( $\mathrm{pH} \mathrm{6.0)}$ in a pressure cooker for $2 \mathrm{~min}$ to retrieve the antigen. The sections were incubated with $3 \%$ hydrogen peroxide solution at room temperature for $20 \mathrm{~min}$ to inhibit endogenous peroxidases and then TBS washed 3 times. Then, $5 \%$ goat serum for closing $20 \mathrm{~min}$, directly dripping primary antibody HIF-1 $\alpha$ (1:250), Bcl-2 (1:250) at $4^{\circ} \mathrm{C}$ overnight. The sections were restored for $40 \mathrm{~min}$ at room temperature. Then, biotin-labeled secondary antibody was added, hatched for $25 \mathrm{~min}$ at $37^{\circ} \mathrm{C}$, and followed by DAB for $30 \mathrm{~min}$. Each step was washed with $0.01 \mathrm{~mol} / 1$ ( $\mathrm{pH} 7.4$ slightly) PBS for 5 min three times. Sections were visualized by using horseradish peroxidase, then counterstained with hematoxylin for $3 \mathrm{~min}$.

Quantitative and qualitative analyses. Each sample was stained under the same conditions, then scanned under a 40x10 Olympus microscope. Ten image fields were randomly selected from each section and then used the Image $\mathrm{J}$ Analysis System (version 1.46, public domain, Image-Processing and Analysis in Java) to measure and analyze the cells. In all, 200 spermatogenic cells were randomly measured in each image. We scored the results according to the grey value of the cells: Grey values of $\sim 80,80 \sim 120,120 \sim 160$, and $\sim 160$ were scores of $0,1,2$, and 4 , respectively. Then, the average score of each cell was calculated.

Western-blot. Testes were washed in precooling normal saline, cut into small pieces, weighed and homogenized with 10 volumes of lysis buffer (containing PMSF) (P0013; Beyotime Biotechnology, Shanghai, China) and then placed on ice for
Table I. Jonhsen score and seminiferous epithelia cells apoptosis index (AI\%) in each group.

\begin{tabular}{lccc}
\hline & Number & Jonhsen score & AI (\%) \\
\hline Group A & 12 & $9.53 \pm 0.18$ & $0.732 \pm 0.215$ \\
Group B & 12 & $7.79 \pm 1.20^{\mathrm{b}}$ & $1.638 \pm 0.640^{\mathrm{b}}$ \\
Group C & 11 & $5.89 \pm 1.38^{\mathrm{b}, \mathrm{c}}$ & $6.259 \pm 2.220^{\mathrm{b}, \mathrm{d}}$ \\
Group D & 10 & $4.00 \pm 1.66^{\mathrm{b}, \mathrm{d}}$ & $10.306 \pm 2.565^{\mathrm{b}, \mathrm{d}, \mathrm{f}}$ \\
\hline
\end{tabular}

Jonhsen score and Seminiferous epithelia cells apoptosis index (\%), AI Significant difference: ${ }^{a} \mathrm{P}<0.05$ with respect to group $\mathrm{A}$; ${ }^{b} \mathrm{P}<0.01$ with respect to group $\mathrm{A} ;{ }^{\mathrm{C}} \mathrm{P}<0.05$ with respect to group $\mathrm{B}$; ${ }^{\mathrm{d}} \mathrm{P}<0.01$ with respect to group $\mathrm{B} ;{ }^{\mathrm{e}} \mathrm{P}<0.05$ with respect to group $\mathrm{C}$; ${ }^{\mathrm{f}} \mathrm{P}<0.01$ with respect to group $\mathrm{C}$. AI, apoptosis index

$30 \mathrm{~min}$. The lysis solution was pureed in a blender, followed by ultrasonication and centrifugation at $4^{\circ} \mathrm{C}$ for $5 \mathrm{~min}$. The BCA Protein Quantitation kit (P0010; Beyotime Biotechnology) was used to determine the protein concentration. Next, $60 \mu \mathrm{g}$ of protein was mixed in the loading buffer and heated at $100^{\circ} \mathrm{C}$ for $5 \mathrm{~min}$. The proteins were separated by $12 \%$ sodium dodecyl sulfa-polyacrylamide gel electrophoresis (SDS-PAGE). The proteins were transferred onto nitrocellulose membranes. The membranes were washed with TBST for 5 min 3 times, blocked with a solution of $0.1 \%$ Tween-20 containing 5\% non-fat dry milk for $2 \mathrm{~h}$, and then incubated with primary antibody at $4^{\circ} \mathrm{C}$ overnight. The membranes were incubated with IgG secondary antibody $(1: 10,000)$ bound horseradish peroxidase for $1 \mathrm{~h}$ at room temperature. The proteins of the bands were imaged using the ECL method. The relative density of the bands was normalized with $\beta$-actin as a control. Software Image $\mathbf{J}$ were used to measure the contents of the proteins.

Statistical analysis. SPSS 19.0 (IBM SPSS, Armonk, NY, USA) statistical software was used for statistical analysis of the measurement data, and the data were expressed as the means \pm standard error of the means. One-way analysis of variance was applied to analyze the data from two groups, an LSD test applied for homogeneity of variance, and a Dunnett T3 test applied for variance heterogeneity. $\mathrm{P}<0.05$ was considered to indicate a statistically significant difference.

\section{Results}

Histological changes. As the VC prolongs, the damage of the seminiferous tubules are more serious. The damage mainly impairs the structure of the seminiferous tubule; There are fewer cell layers of the seminiferous tubules and the distribution of the seminiferous epithelia cells are disorganized. What's more, there are a few sperm in the seminiferous tubules (Fig. 1).

Jonhsen score. The scores of group B, C and D are significantly lower than group A $(\mathrm{P}<0.01)$; As the $\mathrm{VC}$ prolongs, the scores of group B, C and D decrease, but the scores between group $\mathrm{C}$ and $\mathrm{D}$ have no significant differences $(\mathrm{P}>0.05)$ (Table I). All of these indicate that $\mathrm{VC}$ impairs the spermatogenesis of the testis. 
A

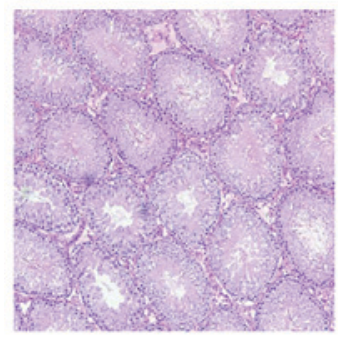

B

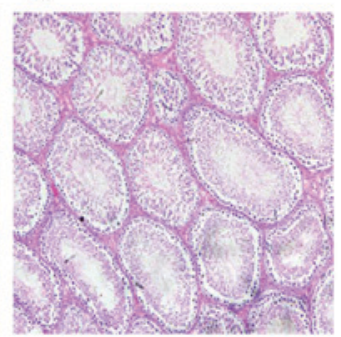

C

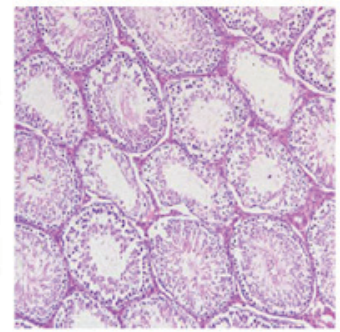

D

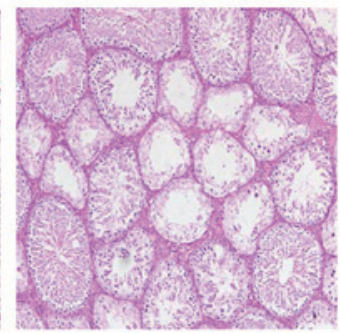

Figure 1. Hematoxylin and eosin staining 10x10 (A) group A (VC 0 day): The basilar membrane of the seminiferous tubule showing the structural integrity and spermatogenic cells distribute from basilar membrane to the lumen. Cells in the seminiferous tubule are hierarchical and orderly, and there are a large number of sperm in the lumen. (B) group B (VC 15 days): The situation is similar to group A. (C) group C (VC 30 days): Partial basilar membrane showing disrupted structural integrity, the number of the cell layers significantly decreased and the cells were disorganized. There are few sperm in the lumen. (D) group D (VC 45 days): The situation is worse than group C. VC, varicocele.

A

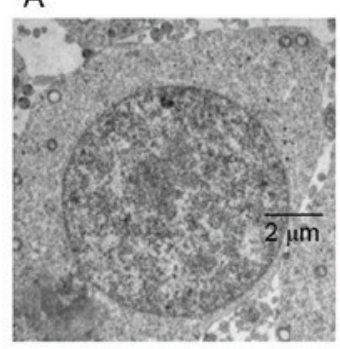

B

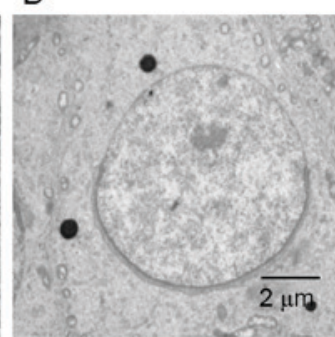

C

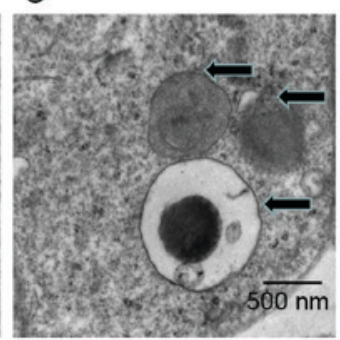

D

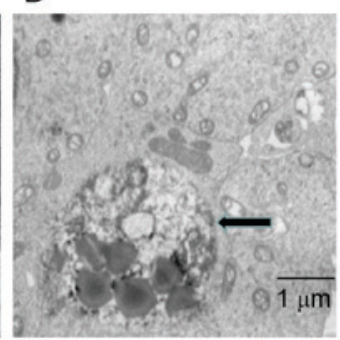

Figure 2. TEM ‘ $\leftarrow$ ' represent lysosomes, autophagosomes or autolysosomes (A) group A (VC 0 day): There are abundant organelles and few autophagosomes, lysosomes, autolysosomes in seminiferous epithelia cells. (B) group B (VC 15 days): Autolysosomes are rare in spermatogenic cells. (C) group C (VC 30 days): Compared to the control group, the number of the autophagosomes, lysosomes, autolysosomes increased significantly in seminiferous epithelia cells. (D) group D (VC 45 days): The situation is similar to group C. TEM, Transmission electron microscopy; VC, varicocele.

A

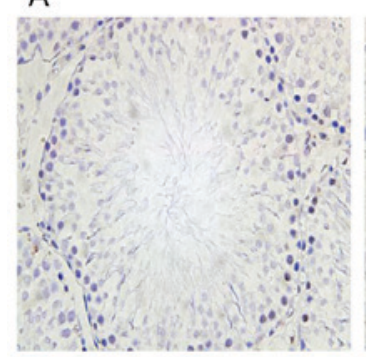

B

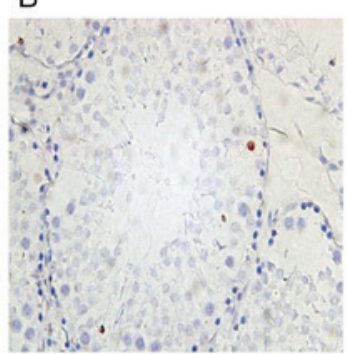

C

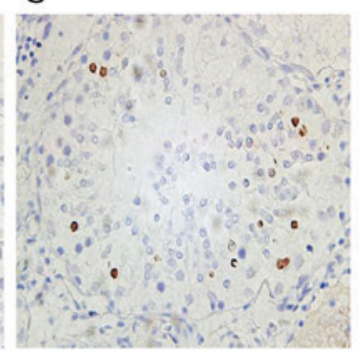

D

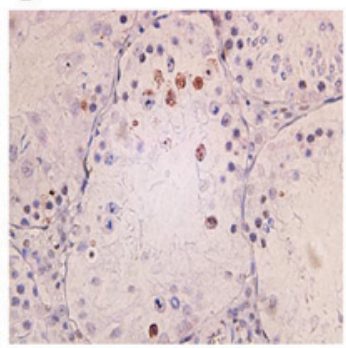

Figure 3. TUNEL staining 40x10 (A) group A (VC 0 day): Apoptotic seminiferous epithelia cells are rarely seen. (B) group B (VC 15 days): There are few apoptotic seminiferous epithelia cells. (C) group C (VC 30 days): The number of the apoptotic seminiferous epithelia cells increased significantly. (D) group D (VC 45 days): The number of the apoptotic seminiferous epithelia cells increased significantly. TUNEL, Terminal-deoxynucleoitidyl Transferase Mediated Nick and Labeling; VC, varicocele.

Transmission electron microscopy. Seminiferous epithelia cells were observed under transmission electron microscopy. In the control group, we found that there were abundant mitochondria in the spermatogenic cells and sertoli cells, and the morphology of the mitochondria were normal. However, as the $\mathrm{VC}$ prolonged, the number of edema mitochondria and autophagosome obviously increased in the spermatogenic cells (Fig. 2).

TUNEL staining. We observed the apoptosis of the seminiferous epithelia cells calculated by TUNEL staining. And found that apoptostic seminiferous epithelia cells were rarely seen in the 30-day VC group, and the number of apoptotic spermatogenic cells increased gradually (Fig. 3). The AI of the control group is approximately $0.73 \%$, but the AI of the 45-day VC group is approximately $10.31 \%$ (Table I). As VC was prolonged, AI of the spermatogenic cells increased rapidly $(\mathrm{P}<0.01)$ (Table I).

Immunohistochemistry. HIF-1 $\alpha$ proteins mainly expressed in the cytoplasm and nucleus of the spermatogenic cells (Fig. 4). As VC was prolonged, the expression of HIF- $1 \alpha$ gradually increased, and average scores among the groups showed a statistically significant difference $(\mathrm{P}<0.01)$ (Table II). Bcl-2 proteins mainly expressed in cytoplasm of the spermatogenic cells (Fig. 5). As VC was prolonged, the expression of Bcl-2 
gradually decreased, and the average scores among the groups showed a statistically significant difference $(\mathrm{P}<0.05)$ (Table III).

Western blot analysis. We measured the proteins related to the HIF-1 $\alpha / \mathrm{BNIP} 3 /$ Beclin1 signaling pathways, and the autophagy molecular markers LC3-II, and other proteins p62, Bcl2 and Bax (Figs. 6 and 7). Compares to the control group, the expression of HIF-1 $\alpha$, BNIP3, Beclin1, and LC3-II/LC3-Iincrease gradually $(\mathrm{P}<0.05)$ (Fig.7A-D). Though the expression of Beclin1 and LC3-II/LC3-Isignificantly increase from the $\mathrm{VC}$ 0-days groups to $\mathrm{VC}-30$ days groups, there are no significant difference between VC-30 and VC-45 $(\mathrm{P}>0.05)$ (Fig. 7C and D). The expression of p62 proteins sharply increases but begins to decrease at VC 45 days (Fig. 7E). What's more the pro-apoptotic protein Bax increase sharply (Fig. 7G) and the anti-apototic protein Bcl-2 decrease gradually (Fig. 7F).

\section{Discussion}

There are many reasons for male infertility caused by VC, such as local temperature rising in testes, hypoxia, oxidative stress and immune abnormalities $(25,26)$. All of these factors will injure the spermatogenesis function of the testis. Through histological observing, as the VC prolongs, the structure of the seminiferous tubules are gradually destroyed; the number of cell layers significantly decreases and the distribution of the seminiferous epithelia cells are disorganized. There are a few sperm in the seminiferous tubule (Fig. 1). Jonhsen score is a good index to evaluate the spermatogenesis function of the testis. We can see, as the VC prolongs, the scores of group A, B, C, D decrease gradually (Table I). These reveal that VC gradually injury the spermatogenesis function of the rats testis.

We examined this tissue through immunohistochemical and Western blot analyses and found that HIF-1 $\alpha$ was positively correlated with VC duration. Other studies $(10,27,28)$ also obtained similar results, indicating that VC can lead to testicular tissue hypoxia, and the degree of hypoxia is highly correlated with VC time. All of these researches indicate that hypoxia may be a important factor for infertility caused by Varicocele. One important role of the hypoxia is to induce HIF-1 $\alpha$, which activates a series of autophagy-related genes, such as BNIP3 (29). As for physiological conditions, Beclin-1 and Bcl-2 form a complex compound leading to inhibit the activandation of the autophagy pathway. Under hypoxia conditions, the expression of HIF-1 $\alpha$ significantly increases, which up-regulates BNIP3. BNIP3 interreacts with Bcl-2 or $\mathrm{Bcl}-\mathrm{XL}$ and eventually forms heterodimer, which will release Beclin-1 from Beclin-1/Bcl-2 complex compound. Then, the free Beclin-1 will activate the autophagy pathway (30).

As a result, HIF-1 $\alpha /$ BNIP3/Beclin-1 signaling pathway is an important way of inducing autophagy under hypoxic conditions. But there are few researches about autophagy in $\mathrm{VC}$ rats testes. Moreover, how does the autophagy be actived still unknown. Therefore, we tested proteins related to this autophagy signaling pathway by Western blot. The expression of HIF-1 $\alpha$, BNIP3, and Beclin-1 significantly increased in the $\mathrm{VC}$ groups compared to the control group $(\mathrm{P}<0.05)$ (Fig. 7A, B and C). This indicates that VC can lead to testicular tissue hypoxia. What's more, hypoxia induces and
Table II. Immunohistochemistry Hif- $1 \alpha$ average score of each seminiferous epithelia cell.

\begin{tabular}{lcl}
\hline & Number & Average score \\
\hline Group A & 12 & $0.645 \pm 0.081$ \\
Group B & 12 & $0.809 \pm 0.071^{\mathrm{b}}$ \\
Group C & 11 & $1.117 \pm 0.098^{\mathrm{b}, \mathrm{d}}$ \\
Group D & 10 & $1.485 \pm 0.120^{\mathrm{b}, \mathrm{d}, \mathrm{f}}$ \\
\hline
\end{tabular}

Hif- $1 \alpha$ average score of each seminiferous epithelia cell, Significant difference: ${ }^{\mathrm{a}} \mathrm{P}<0.05$ with respect to group $\mathrm{A} ;{ }^{\mathrm{b}} \mathrm{P}<0.01$ with respect to group $\mathrm{A} ;{ }^{\mathrm{c}} \mathrm{P}<0.05$ with respect to group $\mathrm{B} ;{ }^{\mathrm{d}} \mathrm{P}<0.01$ with respect to group $\mathrm{B} ;{ }^{e} \mathrm{P}<0.05$ with respect to group $\mathrm{C}$; ${ }^{\mathrm{P}} \mathrm{P}<0.01$ with respect to group $\mathrm{C}$.

Table III. Immunohistochemistry Bcl-2 average score of each seminiferous epithelia cell.

\begin{tabular}{lcl}
\hline & Number & Average score \\
\hline Group A & 12 & $1.963 \pm 0.143$ \\
Group B & 12 & $1.799 \pm 0.102^{\mathrm{b}}$ \\
Group C & 11 & $1.582 \pm 0.108^{\mathrm{b}, \mathrm{d}}$ \\
Group D & 10 & $1.451 \pm 0.177^{\mathrm{b}, \mathrm{d}, \mathrm{e}}$ \\
\hline
\end{tabular}

Bcl-2 average score of each seminiferous epithelia cells, Significant difference: ${ }^{\mathrm{a}} \mathrm{P}<0.05$ with respect to group $\mathrm{A} ;{ }^{b} \mathrm{P}<0.01$ with respect to group $\mathrm{A} ;{ }^{\mathrm{C}} \mathrm{P}<0.05$ with respect to group $\mathrm{B} ;{ }^{\mathrm{d}} \mathrm{P}<0.01$ with respect to group $\mathrm{B} ;{ }^{\mathrm{e}} \mathrm{P}<0.05$ with respect to group $\mathrm{C}$; ${ }^{\mathrm{P}}<0.01$ with respect to group $\mathrm{C}$.

activates the expression of HIF-1 $\alpha$, which may up-regulate the HIF-1 $\alpha /$ BNIP3/Beclin-1 autophagy signaling pathways.

Autophagy is divided into induction, nucleation, elongation, the formation of autophagosome, the fusion of autophagosome and lysosome, and the degradation of substrate. A series of $A T G$ (autophagy related gene) products are involved in the formation of autophagy. Beclin 1 plays an important role in initiating the process of autophagy. At the stage of nucleation, Beclin1 combines with Vps34 and UVRAG to form class III of phosphoinositide 3-kinase (class III PI3K Complex). Class III PI3K Complex III induce the formation of Phosphatidylinositol 3-phosphate (PI3P), which is crucial in the process of autophagy. LC3II distributes inside and outside the surface of the autophagosome membrane as a biomarker for reflecting the level of autophagosome. p62 interacts with LC3II and mediates the ubiquitinated substrates into autophagosome. Moreover, itself and the substrates then degrades in the autophagosome. Therefore, the content of p62 reflects 'autophagic flux'. Through western blot testing, we found that compared with the control group, the expression of Beclin-1 and LC3II is significantly higher in the VC groups $(\mathrm{P}<0.05)$ (Fig. 7C and D). These results reveal that autophagy expresses increasely in $\mathrm{VC}$ rats testes. Furthermore, the expression of p62 proteins sharply increases but begins to decrease at last (Fig. 7E). This indicates that VC may active the autophagy, 


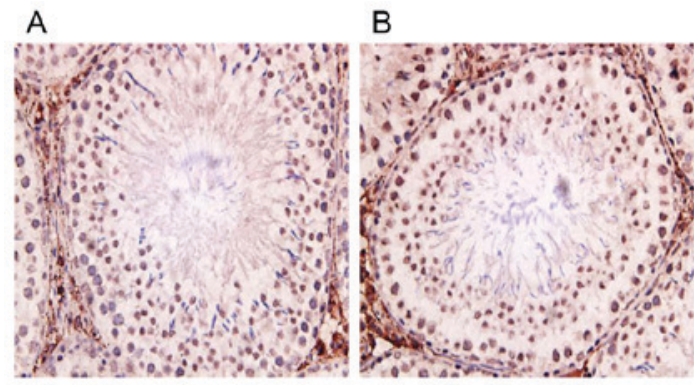

C

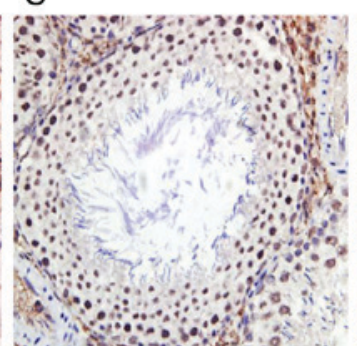

$\mathrm{D}$

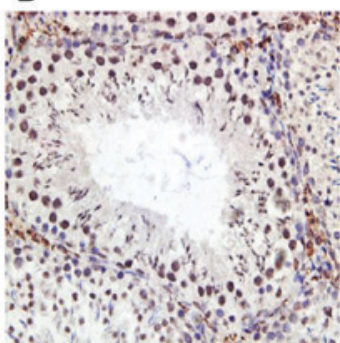

Figure 4. HIF-1 $\alpha$ immunohistochemistry 40x10. HIF-1 $\alpha$ protein is dyed with brown granules, and it is mainly expressed in the nucleus of spermatogenic cells and Sertoli cells. (A) group A (VC 0 day): Brown granules are lowly expressed in the cytoplasm and nuclei of spermatogenic cells. (B) group B (VC 15 days): The expression of brown granules in group B is similar to group A. (C) group C (VC 30 days): Brown granules are highly expressed in nuclei of seminiferous epithelia cells. (D) group D (VC 45 days): Brown granules are highly expressed in the nuclei of spermatogenic cells.

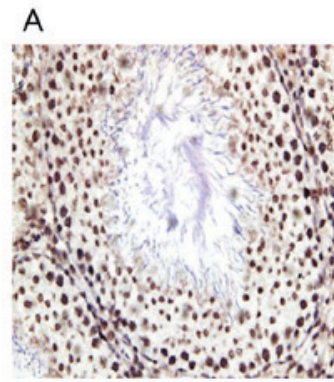

B s.o.

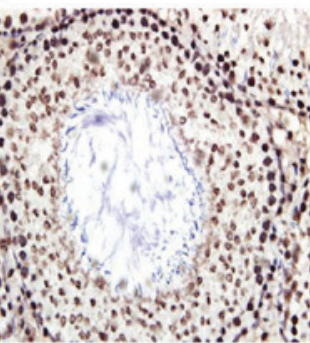

C

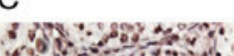

D

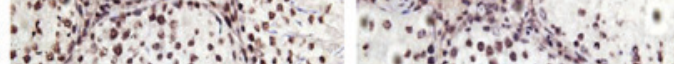

s.t.

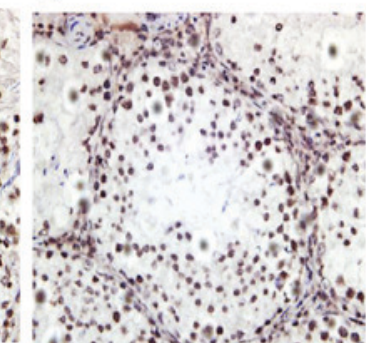

Figure 5. Bcl-2 immunohistochemistry 40x10. Bcl-2 protein is dyed with brown granules, and it is mainly expressed in the nucleus. (A) group A (VC 0 day): Brown granules are highly expressed in the nucleus of seminiferous epithelia cells. (B) group B (VC 15 days): Brown granules are highly expressed in the nucleus. (C) group C (VC 30 days): Brown granules are lowly expressed in the nucleus. (D) group D (VC 45 days): Brown granules are lowly expressed in the cytoplasm of seminiferous epithelia cells.
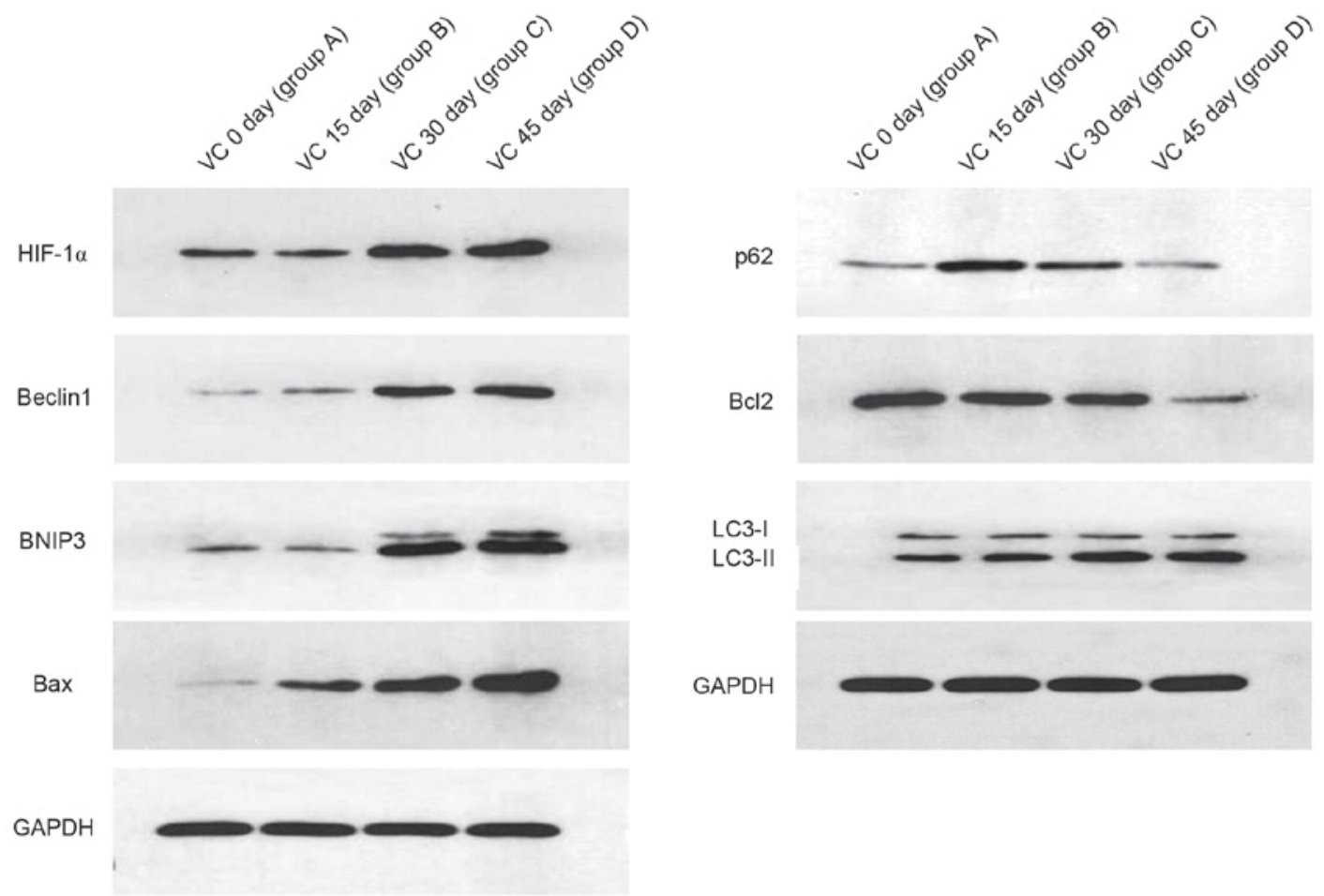

Figure 6. Western blots for HIF-1 $\alpha$, Beclin1, p62, Bc12, Bax, BNIP3, LC3-II, LC3-I, and GAPDH. HIF-1 $\alpha$, hypoxia-inducible factor 1 $\alpha$; Beclin1, BCL2 interacting protein.

but as the VC time prolongs, the 'autophagic flux' decreases, something may effect the autophagy at the late stage of $\mathrm{VC}$.
Transmission electron microscopy is one of the most common and precise methods to observe autophagy in tissue. Moreover, 
A

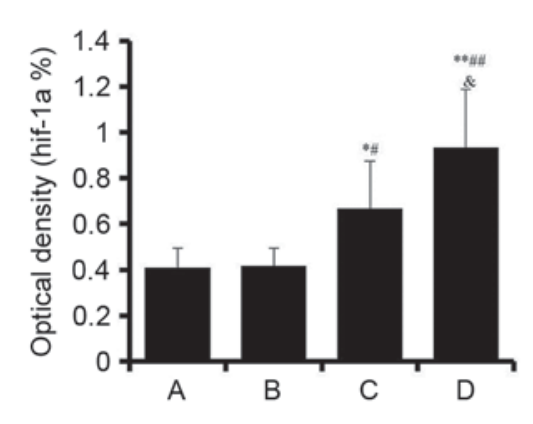

D
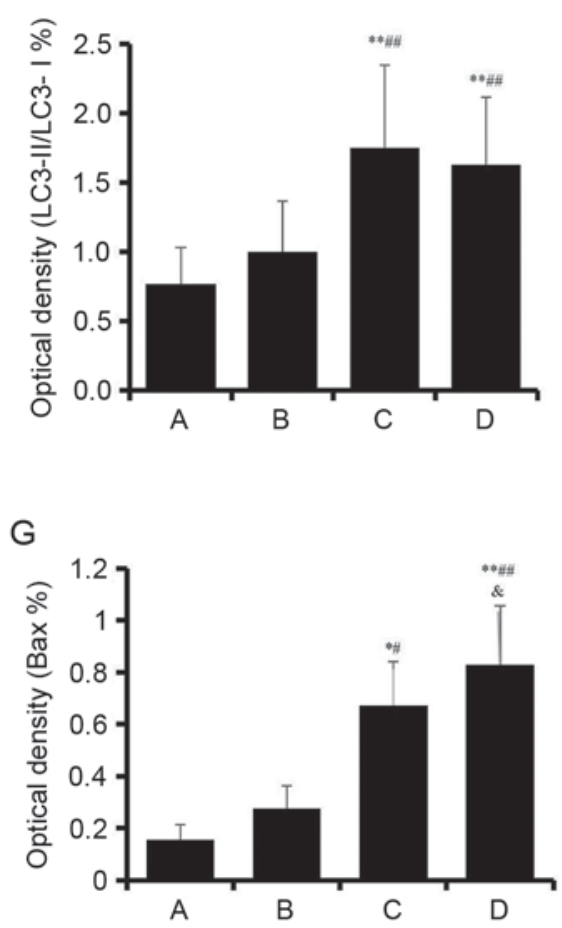

B

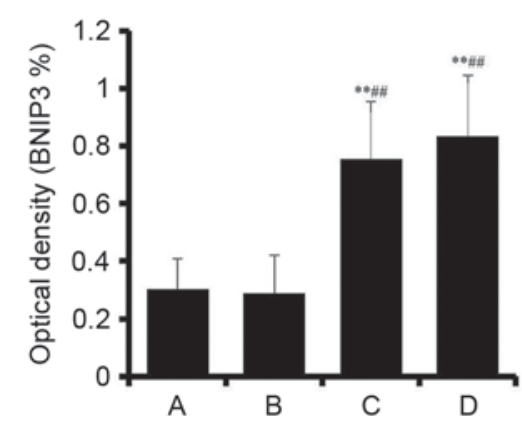

E

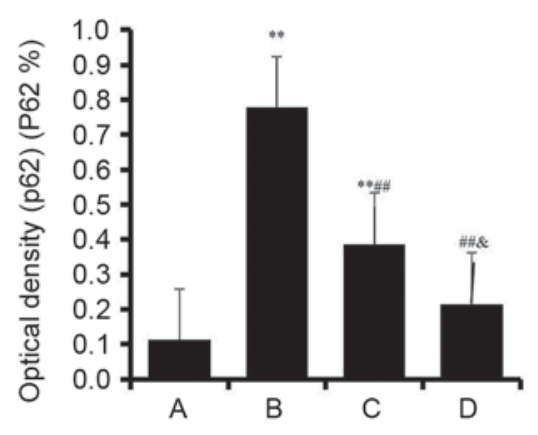

C

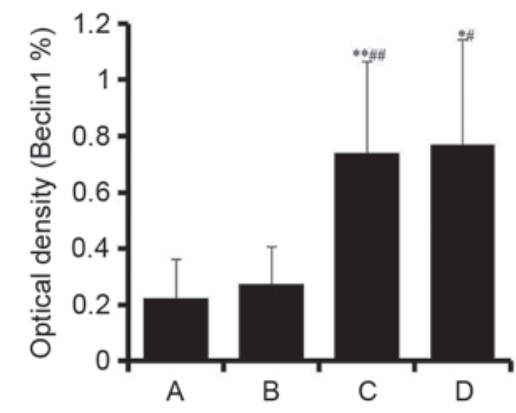

F

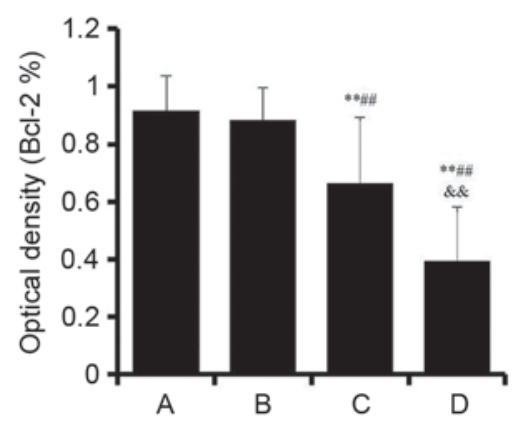

Figure 7. Western blots for (A) HIF-1 $\alpha$, (B) BNIP3, (C) Beclin1, (D) LC3-II/LC3-I, (E) p62, (F) Bcl2, (G) Bax and GAPDH. The intensity of the protein blots was normalized with GAPDH. The data are expressed as the mean \pm SEM. Optical density $(\%)$, significant difference: *P<0.05 with respect to group A; ${ }^{* *} \mathrm{P}<0.01$ with respect to group $\mathrm{A}$; ${ }^{\#} \mathrm{P}<0.05$ with respect to group $\mathrm{B} ;{ }^{\#} \mathrm{P}<0.01$ with respect to group $\mathrm{B}$; ${ }^{\text {\& }} \mathrm{P}<0.05$ with respect to group $\mathrm{C}$; \&\& $\mathrm{P}<0.01$ with respect to group C. HIF-1 $\alpha$, hypoxia-inducible factor $1 \alpha$; Beclin1, BCL2 interacting protein.

under a transmission electron microscope, we found that there were rare autophagosomes, lysosomes, and autolysosomes in the control group. However, the autophagy increased obviously in the 30-day and 45-day groups (Fig. 2). The results of the TEM test support the results of the Western blot test, so we can conclude that VC can lead to the high expression of autophagy in seminiferous epithelia cells.

Apoptosis is an important way to maintain tissue stability; it involves a series of gene activation, transcription and regulation processes. $\mathrm{Bcl}-2$ is one of the most important antiapoptotic proteins in the Bcl-2 family; the four domains gather in tridimensional space and form a hydrophobic groove. The groove can combine with the $\mathrm{BH} 3$ domain of antiapoptotic proteins or $\mathrm{BH} 3$-only proteins, preventing MOMP mediated by the $\mathrm{BH} 3$ domain. Bax is one of a important proapototic proteins in the Bcl-2 family.

Through western blot analysis we found that rats survived VC longer, Bcl2 decreased more obviously; conversely, the expression of Bax increased more obviously. Furthermore, we used TUNEL staining (Fig. 3) to observe the apoptosis of seminiferous epithelia cells in VC rats testes and found that seminiferous epithelia cells AI (apoptosis index, AI) in the 30 and 45-day groups were significantly higher than in the control and VC-15 groups $(\mathrm{P}<0.01)$ (Table I). All the above shows that VC leads to seminiferous epithelia cells apoptosis. Some other researches have reported similar results to ours (10). The apoptosis of seminiferous epithelia cells leads to spermatogenesis impairment, eventually leading to poor quality and quantity of sperm.

Relationship between autophagy and apoptosis. In the early phase of apoptosis, antiapoptotic proteins (such as the Bcl-2) decrease and proapoptosis proteins and $\mathrm{BH}-3$-only proteins increase, which make the beclin1/Bcl-2 or beclin1/Bcl-XL complex depolymerize. Antiapoptotic proteins and proapoptosis proteins form heterodimers. Furthermore, beclin-1 will be freed from the beclin1/Bcl-2 or beclin1/Bcl-XL 
complex, activating autophagy. Under hypoxia conditions, the expression of HIF-1 $\alpha$ increases, which will up-regulate BNIP3 and BNIP3L (BNIP3-like) (31). BNIP3 and BNIP3L are $\mathrm{BH}-3$-only proteins that contain $\mathrm{BH} 3$ domains. They are located in the endoplasmic reticulum, mitochondria and affect apoptosis by regulating the mitochondrial respiration and ROS level. In addition, BNIP3 contains LIR (LC3 interaction region), so it can mediate mitochondrial the autophagy pathway.

As we describe that LC3-II/LC3-Igradually increase from early phage and medium of VC, but there are no difference between VC 30 days groups and VC 45 days groups (Fig. 7D). Moreover, p62 sharply increasely at the early stage of $\mathrm{VC}$, but gradually decrease from VC 15 days, and there are no difference between VC 45 days group and control group (Fig. 7E). It indicates that autophagy is strongly actived at early phage and medium of $\mathrm{VC}$, and may inhibited by some other factors at the late period. Interestingly, We find that $\mathrm{AI}$ and Bax increase gradually, and sharply increase in VC 45 days group (Table I and Fig. 7G). It indicates that the apoptosis of the seminiferous epithelia cells is slightly at early stage of $\mathrm{VC}$, is heavily in the terminal VC. Combined with the changes of LC3-II/LC3-I and p62, we try to hypothesize that early hypoxia does harm to seminiferous epithelia cells at the beginning of VC. The damaged organelles and proteins in the seminiferous epithelia cells activate autophagy. Autophagy can timely remove these damaged substrates to protect cells from secondary lesion. When the cells are severely injured, apoptotic pathways are strongly activated, which will inhibit the autophagy pathway. However, the relationship between apotosis and autophagy is very complicated and still needs further research.

In conclusion, apoptosis and autophagy were firstly observed in the seminiferous epithelia cells in $\mathrm{VC}$ rats testes. HIF-1 $\alpha /$ BNIP3/Beclin1 autophagy signaling pathways may play an important role in the activation of autophagy. In addition, we hypothesize that autophagy may protects seminiferous epithelia cells from apoptosis at the beginning of $\mathrm{VC}$, and apoptosis may inhibit autophagy pathways in later stages. However, the relationship between apoptosis and autophagy is complicated, so the assumption still needs further research. Above all, autophagy may provide a new direction for the diagnosis and treatment of male infertility caused by varicocele.

\section{References}

1. Alsaikhan B, Alrabeeah K, Delouya G and Zini A: Epidemiology of varicocele. Asian J Androl 18: 179-181, 2016.

2. Evers JH, Collins J and Clarke J: Surgery or embolisation for varicoceles in subfertile men. Cochrane Database Syst Rev: CD000479, 2009

3. Baazeem A, Belzile E, Ciampi A, Dohle G, Jarvi K, Salonia A, Weidner W and Zini A, Varicocele and male factor infertility treatment: A new meta-analysis and review of the role of varicocele repair. Eur Urol 60: 796-808, 2011.

4. Rankin EB and Giaccia AJ: Hypoxic control of metastasis. Science 352: 175-180, 2016

5. Wang F, Zhang H, Xu N, Huang N, Tian C, Ye A, Hu G, He J and Zhang Y: A novel hypoxia-induced miR-147a regulates cell proliferation through a positive feedback loop of stabilizing HIF-1 $\alpha$. Cancer Biol Ther 17: 790-798, 2016.

6. Bernardi R, Guernah I, Jin D, Grisendi S, Alimonti A, Teruya-Feldstein J, Cordon-Cardo C, Simon MC, Rafii S and Pandolfi PP: PML inhibits HIF-1alpha translation and neoangiogenesis through repression of mTOR. Nature 442: 779-785, 2006 .
7. Wu H, Huang S, Chen Z, Liu W, Zhou X and Zhang D: Hypoxia-induced autophagy contributes to the invasion of salivary adenoid cystic carcinoma through the HIF-1 $\alpha /$ BNIP3 signaling pathway. Mol Med Rep 12: 6467-6474, 2015.

8. Azad MB and Gibson SB: Role of BNIP3 in proliferation and hypoxia-induced autophagy: Implications for personalized cancer therapies. Ann N Y Acad Sci 1210: 8-16, 2010.

9. Azad MB, Chen Y, Henson ES, Cizeau J, McMillan-Ward E, Israels SJ and Gibson SB: Hypoxia induces autophagic cell death in apoptosis-competent cells through a mechanism involving BNIP3. Autophagy 4: 195-204, 2008.

10. Wang H, Sun Y, Wang L, Xu C, Yang Q, Liu B and Liu Z: Hypoxia-induced apoptosis in the bilateral testes of rats with left-sided varicocele: A new way to think about the varicocele. J Androl 31: 299-305, 2010.

11. Jang H, Kim SJ, Yuk SM, Han DS, Ha US, Hong SH, Lee JY, Hwang TK, Hwang SY and Kim SW: Effects of anthocyanin extracted from black soybean seed coat on spermatogenesis in a rat varicocele-induced model. Reprod Fertil Dev 24: 649-655, 2012.

12. Wu H, Lin J, Liu P, Huang Z, Zhao P, Jin H, Ma J, Wen L and Gu N: Reactive oxygen species acts as executor in radiation enhancement and autophagy inducing by AgNPs. Biomaterials 101: 1-9, 2016.

13. Shoji-Kawata S, Sumpter R, Leveno M, Campbell GR, Zou Z, Kinch L, Wilkins AD, Sun Q, Pallauf K, MacDuff D, et al: Identification of a candidate therapeutic autophagy-inducing peptide. Nature 494: 201-206, 2013.

14. Betin VM and Lane JD: Atg4D at the interface between autophagy and apoptosis. Autophagy 5: 1057-1059, 2009.

15. Martinez J, Malireddi RK, Lu Q, Cunha LD, Pelletier S, Gingras S, Orchard R, Guan JL, Tan H, Peng J, et al: Molecular characterization of LC3-associated phagocytosis reveals distinct roles for Rubicon, NOX2 and autophagy proteins. Nat Cell Biol 17: 893-906, 2015.

16. Hussein MR, Haemel AK and Wood GS: Apoptosis and melanoma: Molecular mechanisms. J Pathol 199: 275-288, 2003.

17. Green DR and Walczak H: Apoptosis therapy: Driving cancers down the road to ruin. Nat Med 19: 131-133, 2013.

18. Orrenius S, Gogvadze V and Zhivotovsky B: Mitochondrial oxidative stress: Implications for cell death. Annu Rev Pharmacol Toxicol 47: 143-183, 2007.

19. Chen HC, Kanai M, Inoue-Yamauchi A, Tu HC, Huang Y, Ren D, Kim H, Takeda S, Reyna DE, Chan PM, et al: An interconnected hierarchical model of cell death regulation by the BCL-2 family. Nat Cell Biol 17: 1270-1281, 2015.

20. Bolat D, Oltulu F, Uysal A, Kose T, Gunlusoy B, Yigitturk G, Turk NS and Turan T: Effects of losartan on experimental varicocele-induced testicular germ cell apoptosis. Andrologia 48: 840-846, 2016.

21. Mostafa T, Rashed L, Nabil N and Amin R: Seminal BAX and BCL2 gene and protein expressions in infertile men with varicocele. Urology 84: 590-595, 2014.

22. Chang FW, Sun GH, Cheng YY, Chen IC, Chien HH and Wu GJ: Effects of varicocele upon the expression of apoptosis-related proteins. Andrologia 42: 225-230, 2010.

23. Turner TT: The study of varicocele through the use of animal models. Hum Reprod Update 7: 78-84, 2001.

24. Johnsen SG: Testicular biopsy score count-a method for registration of spermatogenesis in human testes: Normal values and results in 335 hypogonadal males. Hormones 1: 2-25, 1970.

25. Cho CL, Esteves SC and Agarwal A: Novel insights into the pathophysiology of varicocele and its association with reactive oxygen species and sperm DNA fragmentation. Asian $\mathbf{J}$ Androl 18: 186-193, 2016.

26. Zhao Y, Zhao E, Zhang C and Zhang H: Study of the changes of acrosomal enzyme, nitric oxide synthase, and superoxide dismutase of infertile patients with positive antisperm antibody in seminal plasma. Cell Biochem Biophys 73: 639-642, 2015.

27. Paick JS, Park K, Kim SW, Park JW, Kim JJ, Kim MS and Park JY: Increased expression of hypoxia-inducible factor-1 $\alpha$ and connective tissue growth factor accompanied by fibrosis in the rat testis of varicocele. Actas Urol Esp 36: 282-288, 2012.

28. Goren MR, Kilinc F, Kayaselcuk F, Ozer C, Oguzulgen I and Hasirci E: Effects of experimental left varicocele repair on hypoxia-inducible factor- $1 \alpha$ and vascular endothelial growth factor expressions and angiogenesis in rat testis. Andrologia 49: 2017. 
29. Hsieh DJ, Kuo WW, Lai YP, Shibu MA, Shen CY, Pai P, Yeh YL, Lin JY, Viswanadha VP and Huang CY: 17 $\beta$-Estradiol and/or estrogen receptor $\beta$ attenuate the autophagic and apoptotic effects induced by prolonged hypoxia through HIF-1 $\alpha$-Mediated BNIP3 and IGFBP-3 signaling blockage. Cell Physiol Biochem 36: 274-284, 2015.
30. Lessene G, Czabotar PE and Colman PM: BCL-2 family antagonists for cancer therapy. Nat Rev Drug Discov 7: 989-1000, 2008. 31. Adams JM, Difazio LT, Rolandelli RH, Luján JJ, Haskó G, Csóka B, Selmeczy Z and Németh ZH: HIF-1: A key mediator in hypoxia. Acta Physiol Hung 96: 19-28, 2009. 